\title{
Iniciativas de gestão da clínica empreendidas por enfermeiros em posição estratégica
}

\section{de liderança}

\author{
Clinical management initiatives undertaken by nurses in a strategic leadership position \\ Iniciativas de gestión clínica emprendidas por enfermeras en una posición de liderazgo estratégico
}

Recebido: 20/02/2021 | Revisado: 26/02/2021 | Aceito: 03/03/2021 | Publicado: 11/03/2021

Tiago Modelo Schmitz

ORCID: https://orcid.org/0000-0003-1198-5987 Faculdades Integradas de Taquara, Brasil

E-mail: tiago@ sou.faccat.br

Samanta Andresa Richter

ORCID: https://orcid.org/0000-0002-7506-0126 Pontifícia Universidade Católica do Rio Grande do Sul, Brasil E-mail: samantarichter@sou.faccat.br

Claudia Capellari

ORCID: https://orcid.org/0000-0002-2003-1424 Faculdades Integradas de Taquara, Brasil

E-mail: claudiacapellari@faccat.br

Mariene Araújo Rodrigues Marques

ORCID: https://orcid.org/0000-0002-8720-9899

Universidade Federal de Mato Grosso, Brasil

E-mail: mariene.armarques@gmail.com

Michelly Kim de Oliveira Rosa Guimarães

ORCID: https://orcid.org/0000-0001-8675-664X

Universidade Federal de Mato Grosso, Brasil

E-mail: michellykim.guimaraes@gmail.com

Dagmar Elaine Kaiser

ORCID: https://orcid.org/0000-0001-5652-4653

Universidade Federal do Rio Grande do Sul, Brasil

E-mail: dagmarelainekaiser@gmail.com

Mara Regina Rosa Ribeiro

ORCID: https://orcid.org/0000-0002-7132-3005

Universidade Federal de Mato Grosso, Brasil

E-mail: mrrribeiro10@gmail.com

Gímerson Erick Ferreira

ORCID: https://orcid.org/0000-0002-4039-0205

Universidade Federal de Mato Grosso, Brasil

E-mail: gimersonerick.ufmt@gmail.com

\begin{abstract}
Resumo
No presente estudo, propõe-se a caracterização de iniciativas de gestão da clínica empreendidas por enfermeiros em posição estratégica de liderança. Para isto, foi desenvolvida uma pesquisa descritiva, exploratória, de abordagem qualitativa, entre setembro e outubro de 2018, com participação de oito enfermeiros em cargos de liderança da atenção primária à saúde de um município do Sul do Brasil. Os mesmos responderam a entrevistas audiogravadas, cujos questionamentos focaram abordagens relacionadas aos eixos gestão-assistência-educação nas práticas dos participantes. Após, procedeu-se à análise de conteúdo, modalidade temática. Os resultados ilustram disposições proativas ao alcance de melhores resultados no que tange à gestão das práticas assistenciais, à orientação de ações focadas nas necessidades dos usuários, e ao desenvolvimento profissional da equipe de modo permanente. As iniciativas de gestão da clínica empreendidas por enfermeiros em cargos de liderança revelam amplas oportunidades de disseminação de práticas inovadoras e eficazes nos serviços de saúde, e anuncia o potencial estratégico destes profissionais na condução de processos gerenciais que incitem movimentos em prol da organização em redes com vistas à produção de uma atenção segura, de qualidade, e fundamentada em padrões de excelência.
\end{abstract}

Palavras-chave: Gestão clínica; Enfermeiras e enfermeiros; Capacidade de liderança e governança; Gerenciamento da prática profissional; Gestão de serviços de saúde.

\footnotetext{
Abstract

In the present study, it is proposed to characterize clinical management initiatives undertaken by nurses in a strategic leadership position. For this, a descriptive, exploratory, qualitative research was developed between September and
} 
October 2018, with the participation of eight nurses in leadership positions in primary health care in a municipality in the south of Brazil. The same findings to be identified audio-recorded, questioning focused on the managementassistance-education axes in the participants' practices. Afterwards, content analysis was carried out, thematic modality. The results illustrate proactive dispositions to achieve the best results with regard to the management of care practices, the orientation of actions focused on the needs of users and the professional development of the team on a permanent basis. The clinic management initiatives undertaken by nurses in leadership positions reveal ample opportunities for the dissemination of innovative and effective practices in health services, and announce the strategic potential of these professionals in conducting management processes that incite movements in favor of the organization in networks with with a view to producing safe, quality care based on standards of excellence.

Keywords: Clinical governance; Nurses; Leadership; Professional management; Health services administration.

\section{Resumen}

En el presente estudio se propone caracterizar las iniciativas de gestión clínica emprendidas por enfermeros en una posición estratégica de liderazgo. Para ello, se desarrolló una investigación descriptiva, exploratoria, cualitativa entre septiembre y octubre de 2018, con la participación de ocho enfermeras en puestos de liderazgo en atención primaria de salud en un municipio del sur de Brasil. Los mismos hallazgos se identificarán en audio grabado, cuestionamiento centrado en los ejes de gestión-asistencia-educación en las prácticas de los participantes. Posteriormente se realizó análisis de contenido, modalidad temática. Los resultados ilustran disposiciones proactivas para lograr los mejores resultados en cuanto a la gestión de las prácticas asistenciales, la orientación de acciones enfocadas a las necesidades de los usuarios y el desarrollo profesional del equipo de manera permanente. Las iniciativas de gestión clínica llevadas a cabo por enfermeros en posiciones de liderazgo revelan amplias oportunidades para la difusión de prácticas innovadoras y efectivas en los servicios de salud, y anuncian el potencial estratégico de estos profesionales en la conducción de procesos de gestión que incitan movimientos a favor de la organización en redes con un con miras a producir una atención segura y de calidad basada en estándares de excelencia.

Palabras clave: Gestión Clínica; Enfermeras y Enfermeros; Capacidad de Liderazgo y Gobernanza; Gestión de la Práctica Profesional; Administración de los Servicios de Salud.

\section{Introdução}

As constantes exigências do mundo do trabalho contemporâneo têm impulsionado organizações e serviços de saúde à incorporação de elevados padrões de qualidade, demandando investimentos proativos e empreendedores de seus profissionais, especialmente daqueles que ocupam posição estratégica de liderança, na solução de problemas e apropriação do processo decisório, com maior grau de autonomia (Ferreira, Dall'Agnol, \& Porto, 2016; Dal Molin et al., 2019; Richter et al., 2019). No que diz respeito ao equacionamento e resolutividade das necessidades de saúde das pessoas e populações de maneira equitativa e integral, a gestão de serviços de saúde tem evoluído e desenvolvido modelos propositivos a este fim, e o que se conhece atualmente como gestão da clínica, tem sido amplamente reconhecido como potencial ao incremento de uma clínica segura, compartilhada, de qualidade, e fundamentada em evidências (Gomes et al., 2015; Padilha et al., 2018).

A gestão da clínica consiste em conjunto de tecnologias de microgestão, que visa a qualificação e a efetividade da atenção à saúde, por meio de elevados padrões de qualidade, cujos princípios, fundamentados na indissociabilidade entre gestão, atenção à saúde e educação, favorecem a melhoria contínua dos serviços e das práticas, resultando em ambiente propício à excelência clínica (Gomes et al., 2015; Mendes, 2015; Padilha et al., 2018). Apesar de almejada, a excelência clínica viabilizada por abordagens de gestão da clínica não é garantida, haja vista que depende, dentre outros aspectos, da atuação compartilhada e envolvimento de diversos atores organizacionais na gestão dos processos (Padilha et al., 2018). Nesse escopo, emerge a atuação tática do enfermeiro com vistas à sua implementação, uma vez que este profissional, cada vez mais, tem assumido posições estratégicas de liderança nas organizações e serviços de saúde (Richter et al., 2019), algo que demanda maior engajamento organizacional, autonomia e participação nos processos decisórios, essenciais à garantia de uma assistência segura e de qualidade (Santos et al., 2013).

Especialmente no âmbito da Atenção Primária à Saúde (APS), porta de entrada do sistema de saúde brasileiro, a coordenação dos fluxos de usuários entre os vários serviços das redes de atenção, mediante iniciativas que garantam efetividade das práticas, em orientação às necessidades da população (Mendes, 2015), mostra-se social e cientificamente relevante, por 
constituir espaço favorável à atuação proativa de profissionais em cargo de gestão, e dentre estes o enfermeiro, o que pode favorecer sua autonomia e o desenvolvimento da capacidade de liderança e governança na disseminação de práticas que corroborem com a melhoria da qualidade dos serviços (Santos et al., 2013; Ferreira et al., 2016; Copelli et al., 2017; Dal Molin et al., 2019; Richter et al., 2019).

Em que pese os benefícios da implementação de movimentos em prol da gestão da clínica, não se encontram estudos que enfoquem especificamente tais iniciativas por enfermeiros em cargos de liderança. Esse fato foi constatado em buscas realizadas nas principais bibliotecas eletrônicas e bases de dados virtuais online, a saber: Scientific Electronic Library Online (SciELO); Literatura Latino-Americana em Ciências da Saúde (LILACS); Biblioteca Virtual em Saúde (BVS); National Library of Medicine (Pubmed); e Base de Dados em Enfermagem (BDENF), referentes à última década, utilizando-se os descritores Gestão Clínica, Enfermeiras e Enfermeiros, Capacidade de Liderança e Governança, Gerenciamento da Prática Profissional, Gestão de Serviços de Saúde, e respectivas versões nos idiomas inglês e espanhol.

Acredita-se ser de extrema relevância destacar a importância da gestão da clínica ao alicerçar princípios voltados à produção de intervenções transformadoras, mediante articulação de diferentes saberes e compartilhamento do poder de decisão em prol de uma atenção integral, segura, de qualidade, orientada às necessidades de saúde de pessoas e populações (Gomes et al., 2015; Mendes, 2015; Padilha et al., 2018); contextualizando o reconhecimento que se almeja e direciona à valorização da coordenação dos cuidados de saúde, por meio do papel gerencial do enfermeiro para a articulação e interação de todos os atores e serviços envolvidos na rede. Especialmente no momento atual, em que foram celebrados por meio da campanha mundial Nursing Now os 200 anos da precursora da profissão e protagonista de projetos empreendedores na saúde, enfermeira Florence Nightingale (Cassiani \& Lira Neto, 2018), salientar as iniciativas de gestão da clínica desempenhadas por enfermeiros com funções estrategicamente importantes mostra-se relevante e confere notoriedade às práticas de liderança nos pontos de atenção à saúde.

Nesse sentido, pesquisas relacionadas às iniciativas favoráveis à implementação da gestão da clínica são importantes e necessárias porque, com base em seus resultados, profissionais em posição estratégica de liderança poderão (re)planejar suas ações de maneira mais assertiva, focados na transformação de práticas de atenção, gestão, e educação, com vistas à criação de ambientes propícios à excelência na produção de cuidados em saúde. Em meio às considerações ora apresentadas, faz-se oportuno questionar: Que iniciativas de gestão da clínica são empreendidas por enfermeiros em posição estratégica de liderança em serviços de APS?

Diante disso, o objetivo deste estudo consistiu em caracterizar iniciativas de gestão da clínica empreendidas por enfermeiros em posição estratégica de liderança.

\section{Metodologia}

Trata-se de uma pesquisa de abordagem qualitativa, desenvolvida atendendo-se aos critérios do Consolidated criteria for reporting qualitative research (COREQ). Foi fundamentada em premissas da gestão da clínica, suporte teórico que relacionase, em essência, com o objeto deste estudo. A abordagem qualitativa permitiu um olhar interpretativo do mundo do trabalho quanto às iniciativas de gestão da clínica empreendidas por enfermeiros em posição estratégica de liderança, na caracterização dos fenômenos em termos dos significados que os próprios atores conferem nos cenários (Minayo, 2012).

O estudo foi desenvolvido em serviços de APS de município situado na Região das Hortênsias, Estado do Rio Grande do Sul, Brasil. Nestes, ocupam posição estratégica de liderança quinze enfermeiros, que atuam na coordenação de Unidades Básicas de Saúde, de serviços de Vigilância Sanitária e no Centro de Apoio Psicossocial.

A amostra do estudo deu-se por conveniência, sendo critério de inclusão ocupar posição estratégica de liderança em serviços de APS do município há pelo menos seis meses, partindo da premissa de que estes teriam conhecimento ampliado acerca 
de princípios que configuram a proposta de gestão da clínica. Como critérios de exclusão, consideraram-se afastamentos de qualquer natureza no período de operacionalização da coleta de dados, entre setembro e outubro de 2018. A totalidade de enfermeiros em estudo foi convidada à participação, verificando-se previamente os critérios de elegibilidade. Destes, três participantes recusaram-se à participação, e quatro não atenderam aos critérios.

Para verificar a adequabilidade do instrumento, previamente aplicou-se em teste piloto com dois enfermeiros que ocupam posição estratégica de liderança em seus trabalhos, o que legitimou a empregabilidade do instrumento. A coleta foi integralmente procedida por um único pesquisador, devidamente capacitado para a realização de entrevistas, a fim de atenuar vieses comuns a estudos qualitativos. Os participantes foram abordados em seus respectivos locais de trabalho, com data e horário previamente agendados. Na ocasião, responderam a uma entrevista individual, semiestruturada, gravada e norteada pela seguinte questão: "Fale-me sobre as iniciativas de gestão da clínica empreendidas na APS deste município". Para fins deste estudo, consideraram-se iniciativas de gestão da clínica, aquelas que refletem a atuação com base em melhores práticas clínicas e que são passíveis de planejamento, fundamentada em evidências e indicadores de resultados; descentralização e corresponsabilização das práticas de liderança; e estratégias de melhoria e aperfeiçoamento contínuo, com base nas necessidades de saúde de pessoas, e que convocam ações permanentes de educação em/na saúde; sendo tais considerações orientadoras à condução das entrevistas. A sequência destas ocorreu de modo aleatório. O conteúdo foi gravado em dispositivo media player e, posteriormente, transcrito na íntegra, para garantir a totalidade e fidedignidade das informações. Em seguida, procedeu-se à revisão dos textos por meio de leitura do material transcrito e audição das gravações, simultaneamente, certificando-se de que a essência das informações não fosse modificada, e ajustando em relação à ortografia.

As informações resultantes da coleta de dados foram submetidas à análise de conteúdo do tipo temática (Minayo, 2012), sendo as informações processadas, em termos operacionais, pelas etapas de pré-análise, categorização e tratamento, e interpretação dos resultados. Realizou-se inicialmente a leitura flutuante do conteúdo transcrito, com vistas à checagem da fidedignidade das informações transcritas em relação aos áudios das gravações. Após leituras repetidas, de caráter analítico, as ideias centrais foram sistematizadas em unidades de sentido, conforme homogeneidade e pertinência, constituindo assim o corpus de análise que possibilitou a categorização dos dados. A categorização deu-se por aglutinação, conforme semelhança temática, sendo estruturada e sustentada em interpretações por referenciais de gestão da clínica (Gomes et al., 2015; Mendes, 2015; Padilha et al., 2018). Na apresentação dos resultados, os excertos dos relatos foram editados em observância às normas gramaticais e/ou acrescidos de termos em colchetes, no intuito de facilitar a compreensão das falas, contudo, sem incorrer na alteração da essência/sentido do conteúdo.

Os preceitos éticos e legais estabelecidos na Resolução 466/2012 do Conselho Nacional de Saúde foram cumpridos, sendo iniciada a coleta de dados somente após aprovação do projeto de pesquisa pelo Comitê de Ética em Pesquisa das Faculdades Integradas de Taquara, conforme parecer consubstanciado: 3.479.049, e registro CAAE: 94110918.8.0000.8135 na Plataforma Brasil. Todos os participantes assinaram o Termo de Consentimento Livre e Esclarecido (TCLE), em duas vias de mesmo teor, também assinadas pelos pesquisadores. Ademais, em preservação ao anonimato dos participantes, no final dos excertos, foi acrescida a notação "E", que representa "enfermeiro", seguido de um número arábico, que indica a sequência cronológica de realização da entrevista.

\section{Resultados}

O estudo contou com a participação de oito enfermeiros em cargos de gestão, sendo a amostra predominantemente feminina (75\%), com idade entre 35 e 55 anos (87,5\%), nível de especialização lato sensu (62,5\%) e experiência de trabalho como enfermeiro por período superior a oito anos (75\%). Os relatos dos profissionais permitiram identificar iniciativas de gestão da clínica no exercício de suas posições estratégicas de liderança na APS, apreendidas em três categorias temáticas: Organização 
da atenção com base nas necessidades de saúde e padrões gerenciais; Articulação e corresponsabilização entre atores, práticas e níveis de cuidado; e Ações educativas permanentes em reorientação às práticas de cuidado.

\subsection{Organização da atenção com base nas necessidades de saúde e padrões gerenciais}

Evidenciaram-se iniciativas que contextualizam o potencial de liderança dos enfermeiros em cargo de gestão, mediante visão ampliada ao organizar a atenção à saúde de acordo com as necessidades das pessoas e coletivos, com vistas à integralidade do cuidado:

Não tem como fazer gestão sem conhecer as necessidades da população. Estas necessidades são levantadas de duas formas: indicadores quantitativos e análise da situação de saúde, através de ferramentas de gestão. (E1)

Consigo avaliar se os resultados foram alcançados [...]: demanda dos próprios usuários, índices baixos de patologias, processo de trabalho dos profissionais, repasse de recursos financeiros, verbas vindas do Ministério da Saúde [...] relatórios de produção que servem para avaliar, analisar e comparar a produção de toda a minha equipe por mês. (E3)

Não tem como trabalhar sem conhecer um pouquinho do usuário, sem entender as queixas, por ser UBS eles vêm muito aqui, eles não vêm apenas para receber uma medicação ou consultar, vêm para conversar contigo falar dos problemas. (E5)

Acompanho o usuário desde antes do nascimento, até a idade avançada. Assim conheço muito bem meus usuários, faço visita domiciliar, conheço a família, a necessidade dela. Se não aderem a algum tratamento, faço busca ativa. (E8).

Priorizamos aquele usuário com necessidades especiais, dificuldades de locomoção, patologias específicas, restritos ao leito, acamados, levar a assistência até ele. (E2).

$\mathrm{Na}$ análise dos excertos, foram mencionadas ainda, iniciativas que ilustram o emprego de indicadores gerenciais em prol de melhorias dos processos, resultados e práticas de cuidado:

Nos casos em que há índice elevado de alguma patologia, por exemplo, reforço a prevenção, orientação, busca ativa, educação em saúde, juntamente com a equipe. (E3)

Faz todo mês um levantamento de dados, número de pacientes, diagnósticos, altas, internações... A gente fecha esse relatório e faz uma reunião geral. (E7)

\subsection{Articulação e corresponsabilização entre atores, práticas e níveis de cuidado}

No que concerne à atuação profissional na APS, apreenderam-se iniciativas que expõem investimentos dos enfermeiros em posição estratégica de liderança na corresponsabilização de coletivos estratégicos ao cuidados, nos diversos pontos da rede de atenção:

Não fazemos nenhuma ação de cuidado sozinhos, tenho sempre em vista metas e objetivos partilhados com a equipe, conversado com o usuário, para depois desenvolvê-los. (E3)

A resolução dos problemas com diálogo, trabalho em equipe, aqui o cuidado é compartilhado e sempre funciona [...] a gente discute as ações a serem desenvolvidas. Aqui é muito valorizada a participação de todos. (E7)

[...] analisar a situação de saúde, estudar os protocolos já existentes [...] fazer reuniões [...], quem vai fazer parte de 
todo o processo: hospitais, unidades de saúde, laboratórios, [...] e a partir disso, fazer um projeto municipal. (E1)

Tudo começa pelo acolhimento, depois disso a gente atende o usuário, e presta o encaminhamento necessário [...] para outros lugares da rede. (E5)

Trabalho na organização de todos os processos de trabalho, agendamento de fluxo de serviço, desde a hora que o usuário chega [...] organizar a agenda para o fluxo ser melhor, todo o olhar do serviço, tenho procurado fazer sempre conversando com os profissionais [...] visando que o serviço fique fluido e que o usuário saia satisfeito. (E6)

Ao explanar considerações dos participantes acerca de suas iniciativas de gestão da clínica, os depoimentos permeiam estratégias que valorizam e articulam saberes e práticas dos diversos atores envolvidos nas ações de cuidado, com vistas ao equacionamento de demandas do cotidiano:

São várias áreas de formação, então a gente respeita muito a opinião do outro colega. Toda e qualquer ação que não seja rotina é discutida entre a equipe antes de ser implementada. Toda equipe assume a responsabilidade pelo usuário. (E7)

Temos grupos diversos, faço consultas de enfermagem, acompanhamento dos usuários que vêm aos grupos, deixo a unidade aberta para os usuários [...] Tenho um grupo de whatsapp com a equipe para discutir casos específicos. Tenho grupo de hiperdia presencial e no whatsapp pois melhora o vínculo entre os profissionais e os usuários [...] As visitas domiciliares aproximam muito a relação entre os profissionais e a comunidade[...] também, intercalo as consultas com a médica. Temos reunião de coordenação mensalmente, reunião da Estratégia de Saúde da Família quinzenalmente, e temos uma ligação forte com a vigilância. (E8)

Juntamente com toda a equipe, realizamos grupos de apoio de acordo com cada tipo de necessidade encontrada na área. (E3)

\subsection{Ações educativas permanentes em reorientação às práticas de cuidado}

Nos depoimentos, foram constatadas iniciativas que elucidam a importância atribuída às ações de desenvolvimento profissional da equipe, de modo continuado e permanente, como meio estratégico de solução de problemas e reorientação das práticas:

A gente senta com a pessoa que tem mais experiência, sempre fazendo essa troca. Assim como ocorre com o tratamento da sífilis, por exemplo. Todos precisam saber, o técnico, o enfermeiro, a recepção, dentro do que lhes compete. (E4)

A gente sempre procura fazer cursos, usamos muito o AVA-SUS [Ambiente Virtual de Aprendizagem do SUS], definimos na reunião de equipe quem vai fazer, depois essa pessoa repassa para os demais. (E7)

Consigo identificar as necessidades por meio de observação no dia a dia, no processo, no fluxo de trabalho, pela supervisão da minha equipe, analisando o processo de trabalho. Identificando suas necessidades, recebem capacitações [...] para saber lidar com os problemas. (E3)

A equipe traz, ou levamos, ocorre uma troca, nada é feito de cima para baixo, e sim uma troca. Se alguém errou, ok. Como podemos fazer daqui pra frente? (E6)

Nas reuniões entre equipes acontecem capacitações, sempre discutimos sobre algo novo, dúvidas, sobre os casos mais 
complicados da semana, como poderíamos ter feito. (E8)

Todos os profissionais que trabalham aqui, participam das reuniões de equipe, e na medida do possivel a gente ouve todo mundo, porque todo mundo sempre tem algo a contribuir [...] para poder ouvir todas as áreas e assim poder criar ações efetivas. (E2)

\section{Discussão}

Nos excertos apresentados é possível apreender que iniciativas de gestão da clínica demandam planejamento, sistematização, articulação, corresponsabilização e comprometimento dos enfermeiros entrevistados, direcionando estes profissionais à resolução de problemas vivenciados cotidianamente em suas funções estratégicas nos serviços de APS, algo que parece estar sendo implicitamente incorporado e que corrobora para o desenvolvimento e visibilidade da profissão no que tange à assunção de cargos de liderança. Tais iniciativas coadunam com a literatura em relação à atuação de enfermeiros aptos a assumirem posição de liderança na APS, face às novas configurações no seu processo de trabalho e que, além de suscitar novas modelagens de produção do cuidado (Galavote et al., 2016), requer envolvimento, comprometimento, responsabilidade, tomada de decisões e comunicação assertiva na promoção de cuidado e bem estar dos coletivos, servindo de inspiração para seus colaboradores e transformando o trabalho neste nível de cuidado (Mattos \& Balsanelli, 2019).

No que tange à organização da atenção com base nas necessidades de saúde e padrões gerenciais, são referidas iniciativas que expressam o empenho destes enfermeiros na execução de ações que resultem na qualificação da assistência prestada e norteiem a atenção à saúde. Assim, iniciativas de gestão da clínica possibilitam a qualificação dos serviços de saúde com foco nas demandas dos próprios usuários e no uso estratégico de instrumentos, especialmente diretrizes clínicas. Tais instrumentos subsidiam o processo de melhoria contínua na organização da assistência, direcionando o raciocínio clínico e corroborando para a tomada de decisões sobre as necessidades identificadas e dirigidas aos cuidados para usuários, trabalhadores, famílias e coletivos (Cavalcanti, Oliveira Neto \& Sousa, 2015).

Nessa perspectiva, alguns excertos denotam que a adesão a princípios para a gestão da clínica resultou em incremento à qualidade da assistência nas práticas para a gestão do cuidado, na medida em que os enfermeiros exercem o trabalho gerencial para além de questões hierárquicas, recorrendo a indicadores epidemiológicos e de produção, análise da situação de saúde, relatórios de produção, dentre outros recursos essenciais à tomada de decisão gerencial. Além disso, possivelmente, as práticas para a gestão do cuidado vão além da qualificação das ações, e também beneficiam o próprio exercício laboral do enfermeiro, sobretudo por reforçar sua autonomia e potencial estratégico no gerenciamento de recursos, mobilização de pessoal, planejamento, organização e avaliação de ações, tão fundamentais à melhoria dos serviços e das práticas de cuidado (Oliveira, Toso \& Matsuda, 2018).

Percebe-se que as iniciativas empreendidas por estes profissionais são centrais na implementação da gestão da clínica, não só por ampliar de modo promissor os espaços de atuação e visibilidade do enfermeiro (Ferreira et al., 2016), como também por endossar o potencial estratégico do enfermeiro na condução de pessoas e processos (Richter et al., 2019). Faz-se importante, portanto, que iniciativas de gestão da clínica sejam empreendidas por enfermeiros em funções estratégicas de liderança, no intuito de qualificar as práticas de APS, pois corresponde, inclusive, ao desafio da Organização das Nações Unidas, que prima pela transformação do mundo a partir dos Objetivos do Desenvolvimento Sustentável, favorecendo a assunção e contribuições da Enfermagem na defesa do cuidado em saúde (PAHO, 2018). Cumpre salientar as notações de participantes que entendem como iniciativas da gestão da clínica, práticas de cuidados proporcionadas de forma humanizada e com equidade. Nesses termos, vale destacar a coesão entre os achados e o proposto pelos referenciais adotados à sua forma de interpretação (Gomes et al., 2015; Mendes, 2015; Padilha et al., 2018), em virtude da recomendação de que a atenção seja orientada às necessidades de saúde das 
pessoas e coletivos, por meio da transformação de práticas de atenção, gestão e educação, princípio que corresponde à complexidade das ações de saúde, abrangendo as dimensões biológica, psicossocial e cultural, assim como a integralidade do cuidado, com vistas ao atendimento igualitário e equitativo.

Sob esse aspecto, uma das falas mostra-se condizente com perspectivas apresentadas na literatura (Mendes, 2015; Padilha et al., 2018), pois aborda nas iniciativas de gestão da clínica estratégias para ordenação da atenção à saúde através de ações orientadas pelas necessidades de saúde, proporcionando melhorias assistenciais contínuas e resolutivas. Esse é um dado a ser destacado porque, na realidade brasileira, especialmente no âmbito da APS, em que as condições de trabalho mostram-se inadequadas, com orientação de trabalho curativista e individual (Lanzoni, Meirelles \& Cummings, 2016), faz-se necessário superar a fragmentação dos processos de trabalho, das relações entre os profissionais e da compreensão de saúde (Galavote et al., 2016), mediante emprego de estratégias que induzam novas formas de conviver e de se relacionar coletivamente, favorecendo o cuidado empreendedor (Backes et al., 2020) e a mobilização propositiva de recursos em prol de melhores práticas (Ferreira $e t$ al., 2016; Dal Molin et al., 2019; Richter et al., 2019).

O emprego de padrões gerenciais visando melhores resultados e práticas também remete aos princípios do referencial (Mendes, 2015; Padilha et al., 2018), constituindo-se fator importante à orientação do trabalho gerencial, fundamentado em resultados que agregam valor à saúde e à vida. A busca por melhores estratégias, mediadas pela utilização de indicadores gerenciais, perfaz iniciativas que se relacionam à qualificação dos serviços de APS, como denotam os verbetes de alguns enfermeiros deste estudo. Nesse sentido, a gestão da clínica parece ser um aporte para monitorar a eficácia dos serviços, colaborando com a efetividade que exerce na utilização dos recursos, visando melhores padrões de cuidados.

Porém, atribuir a simples utilização de indicadores à implementação da gestão da clínica pode ser imaturo e até contraproducente, já que o emprego destes resultados deve garantir a melhoria da eficiência e efetividade da atenção, cabendo, portanto, a orientação por padrões que agreguem valor à saúde e à vida (Padilha et al., 2018). Considera-se que tal postura corrobora com o referencial de gestão da clínica, que compreende um movimento de transformação da gestão convencional de meios para a microgestão dos fins, em processo que valoriza a intimidade do encontro clínico (Mendes, 2015). Tal perspectiva é corroborada em estudo que visou a produção de artefatos para o cuidado de usuários com lesões crônicas de pele na APS, e constatou que o movimento de implementar princípios para a gestão da clínica é indutor de melhores práticas clínicas no encontro com o serviço (Cordova et al., 2020).

Nessa perspectiva, conjectura-se que, no âmbito da APS, ações gerenciais propositivas e empreendedoras, que ultrapassem o cumprimento de protocolos e contribuam para o desenvolvimento social sustentável (Ferreira et al., 2016; Pereira \& Oliveira, 2018; Dal Molin et al., 2019; Richter et al., 2019; Backes et al., 2020), são necessárias para o alcance de princípios para a gestão da clínica. Isso incorre, inclusive, pelo deslocamento da gestão dos meios para a gestão dos fins, a qual mobiliza os enfermeiros em posição de liderança a sentirem-se corresponsáveis pelos serviços e a fomentar a corresponsabilização de outros atores envolvidos na produção de cuidados em saúde, influenciando, tecendo articulações e estimulando a participação ativa nas decisões estratégicas e operacionais na APS.

Outra iniciativa elencada pelos participantes refere-se justamente à articulação e corresponsabilização entre atores, práticas e níveis de cuidado, fenômeno complexo que abrange a definição e pactuação da responsabilidade, mecanismos de cooperação e articulação de recursos e processos entre atores e instituições envolvidos na rede de atenção à saúde (Padilha et al., 2018). Frente a tais iniciativas, há de se considerar funções estrategicamente importantes, tais quais as desempenhadas por enfermeiros deste estudo, na implementação de princípios para a gestão da clínica, já que estes profissionais comumente têm assumido cargos de liderança nos serviços de saúde, e, nesse sentido, têm a oportunidade ímpar de conduzir a efetivação de políticas públicas e disseminar práticas inovadoras de atenção multiprofissional.

Neste estudo, alguns participantes ratificam a importância de sua atuação enquanto profissional que ocupa posição 
estratégica de liderança nos serviços de saúde, na valorização do trabalho colaborativo e solução de problemas da prática em APS como iniciativa advinda da gestão da clínica no cenário investigado. Compatível com movimentos que intentam a gestão da clínica, a prática interprofissional colaborativa é classicamente reconhecida como prática focada nos usuários e que, por sua configuração de forma colaborativa e em parceria com usuários, trabalhadores, famílias e coletividades, orienta-se às necessidades de saúde, de forma integral (Souza et al., 2016; Peduzzi \& Agreli, 2018).

O exercício do trabalho em equipe interprofissional e colaborativo na APS é outro fator que pode guardar estreita interface com a gestão da clínica, por se relacionar diretamente com qualidade da atenção à saúde, segurança e satisfação dos indivíduos, sendo a enfermagem uma das profissões mais citada nos estudos sobre a temática (Brandt et al., 2014), o que endossa o papel coordenador do enfermeiro na articulação de atores e serviços integrados na rede, tal como apresentado pelos participantes deste estudo. Por isso, mostra-se essencial à implementação da gestão da clínica o papel desempenhado por profissionais em cargo de liderança, e, nesse sentido, a função nuclear de mediação do enfermeiro que ocupa essa posição, visto que, de acordo com a literatura (Souza et al., 2016), este profissional tem intermediado a articulação entre diversos profissionais e serviços na produção do cuidado, contribuindo para a atenção centrada no indivíduo e rompendo com a hegemonia do poder uniprofissional na organização do serviço, respeitando diferentes saberes e potencialidades e estimulando o cuidado compartilhado entre profissionais, pessoas sob cuidado, famílias, comunidade e gestores (Padilha et al., 2018).

Os participantes deste estudo sugerem que iniciativas da gestão da clínica em APS têm relação com a gestão compartilhada, corresponsável e estruturada em redes, a qual pode ter facilitado as ações necessárias para a integração de processos e serviços em suas experiências gerenciais na articulação e corresponsabilização entre os diversos atores envolvidos no cuidado, com seus diferentes saberes e práticas, e do envolvimento de diferentes níveis de atenção na qualidade da assistência. Esse dado corrobora com a literatura (Peduzzi \& Agreli, 2018) ao destacar a necessidade de integração e colaboração que permeia o desejo de profissionais que trabalham juntos em prover melhor atenção à saúde e/ou de modo mais abrangente no cenário das RAS e territórios, mediante colaboração em rede intersetorial e com os coletivos.

Observa-se nesta categoria temática que o cenário investigado parece ser legitimado pela articulação de diferentes saberes e práticas na proposição de intervenções na APS. Isso, possivelmente, deu-se em função das próprias diretrizes da gestão da clínica que, entre outras, impera que o serviço promova uma nova clínica: centrada na pessoa, na família e na comunidade; exercida mediante novas formas de encontro clínico e relações entre a atenção especializada e a APS; mediada pelo equilíbrio entre a atenção presencial e a distância, bem como pelo fortalecimento do autocuidado apoiado; implementada a partir de planos de cuidados interdisciplinares, permeados por atenção colaborativa, multi e interprofissional, em ato (Mendes, 2015).

Sabidamente, os serviços de APS são responsáveis pelo atendimento à maior parte da população brasileira que necessita de cuidados primários (Mendes, 2015; Galavote et al., 2016). Nesse sentido, faz-se importante que os profissionais em posição de liderança nestes serviços estejam preparados para atender a demanda de forma qualificada. De acordo com os participantes deste estudo, essa condição pode ser viabilizada pela inúmeras articulações e estratégias que se estabelecem para criar ações que sejam efetivas.

Esta perspectiva foi constatada em proposta de intervenção (Pinto Filho \& Lima, 2020) que identificou que os profissionais de saúde, para desenvolver práticas orientadas às reais necessidades das pessoas, de forma integral e articulada com os diferentes níveis de atenção em saúde, precisam exercitar o pensamento crítico, proativo e empreendedor ao analisar os problemas da sociedade e buscar soluções para os mesmos. Tal concepção endossa o movimento para a gestão da clínica, reafirmando que tal abordagem não se reduz a ações técnicas, diagnósticas, terapêuticas e procedimentais, e que, embora sejam essenciais à efetivação da clínica, prescindem de mecanismos inovadores de gestão, capazes de solucionar problemas de saúde de determinada população, decorrentes de sistemas fragmentados vigentes.

Cabe considerar outro aspecto, constante em um dos depoimentos: a necessidade de articular profissionais de diferentes 
áreas em implementação de ações de cuidado, por serem deficitários em relação à humanização no atendimento. Isso é importante, pois salienta a elaboração de planos de cuidados orientados para as necessidades de saúde dos coletivos, conforme destacou um dos enfermeiros participantes, fazendo com que as ações multiprofissionais e interdisciplinares coadunem com a natureza complexa dos problemas enfrentados no território.

Em que pese às considerações anteriores, cabe reconhecer a relevância de estudos sobre gestão da clínica, empreendidos no contexto do trabalho assistencial hospitalar, e que apontam que sua implementação favorece a organização dos processos de cuidado, podendo impactar positivamente na qualidade do atendimento e no acesso aos serviços (Anschau et al., 2016; Anschau et al., 2017). Pesquisa que monitorou indicadores hospitalares por período de seis meses, após dois meses de implementação de estratégias de incorporação da gestão da clínica, evidenciou maior acesso e qualidade dos cuidados, em relação ao mesmo período do ano anterior, comprovando diminuição das transferências internas e da taxa de mortalidade (Anschau et al., 2017). Somado a isso, outra investigação evidenciou o aumento de altas para o domicílio em relação ao período anterior, aumento no índice de rotatividade da unidade de leitos de retaguarda, e diminuição no tempo médio de permanência (Anschau et al., 2016).

Ante ao exposto na realidade hospitalar, cumpre a reflexão de que o panorama da APS brasileira é peculiar e complexo porque enfrenta "problemas velhos" como "novos desafios", e que precisam ser solucionados progressivamente para avançar em direção à sua qualificação (Cavalcanti, Oliveira Neto \& Sousa, 2015). Nesse sentido, o pensamento estratégico, crítico e reflexivo, empreendido por enfermeiros em cargos de liderança, voltado à transformação de contextos reais do trabalho em saúde, parece mostrar-se essencial à implementação da gestão da clínica na APS. Além disso, contribui para atender à recomendação do relatório "O Estado da Enfermagem no Mundo" (WHO, 2020), que indica a necessidade de ampliação de postos de trabalho para enfermeiros líderes, fortalecendo sua contribuição aos sistemas de saúde. É o que explicitam os participantes, no tocante aos resultados obtidos que conformam a categoria temática ações educativas permanentes em reorientação às práticas de cuidado.

Os excertos pertinentes à categoria são ilustrativos e corroboram com o princípio de que trata a educação de pessoas e da organização (Padilha et al., 2018), processo cultural articulado de educação permanente e de avaliação contínua que envolve todos os âmbitos do trabalho em saúde, na perspectiva de organização que se transforma. Iniciativas assim, desempenhadas por enfermeiros em posição de liderança, podem contribuir para que demais profissionais da APS sintam-se mais pertencentes e engajados com os propósitos do serviço, associando-se mais fortemente, à construção da capacidade resolutiva do serviço, conforme propõe a gestão da clínica (Mendes, 2015).

Vale ressaltar que na realidade referida, ações educativas parecem ser vivenciadas rotineiramente pela maioria dos profissionais, convocando a utilização de estratégias que favorecem a implementação da gestão da clínica, tais como: trocas, capacitações, disseminação de informações e aprendizados, supervisão, práticas multiprofissionais e interdisciplinares, problematização, busca de soluções e reflexões coletivas. Novamente, os achados apontam para contribuições de tais iniciativas por intermédio de enfermeiros líderes da APS, e, portanto, revelando-se relação profícua, visto que a educação é elo crucial à sua implementação, sendo indutora da melhoria da qualidade na medida em que potencializa o compartilhamento de ideias e mudanças das práticas, e propicia aprendizagens e desenvolvimento profissional qualificado (Gomes et al., 2015), oportunizando com isso, melhor gestão do cuidado pelas práticas de liderança conduzidas pelo enfermeiro (Oliveira, Toso \& Matsuda, 2018). O potencial transformador do enfermeiro em posição estratégica de liderança na APS é reconhecido em diversos estudos (Lanzoni, Meirelles \& Cummings, 2016; Cassiani \& Lira Neto, 2018; Mattos \& Balsanelli, 2019; Richter et al., 2019; Barros et al., 2021) e nesta condição, este profissional assume posicionamento transformacional, algo que favorece a melhoria da eficácia e da qualidade da atenção à saúde e é movido a superar o viés gerencialista, sem a devida reflexão crítica, e orientar-se por padrões de excelência na busca de melhores resultados.

Destaca-se, em diversos excertos, a reorientação das práticas decorrente das trocas que se estabelecem no encontro com 
o serviço, com maior valorização dos saberes prévios, e tomando os desafios e os erros como disparadores da aprendizagem, resultados que na óptica do objeto deste estudo favorecem o diálogo reflexivo entre as iniciativas da gestão da clínica e as informações acerca da realidade (Gomes et al., 2015; Mendes, 2015; Padilha et al., 2018). Essa lógica de trabalho em equipe identificada nos depoimentos, remete a elementos constitutivos do trabalho interprofissional, quais sejam: comunicação, respeito mútuo, reconhecimento do trabalho do outro, e o conflito como catalisador de integração e colaboração entre profissionais (Souza et al., 2016), algo que reafirma a potencialidade do enfermeiro em ocupar posições de liderança, visto que a formação e prática interprofissional, de acordo com a literatura (Brandt et al., 2014; Souza et al., 2016; Peduzzi \& Agreli, 2018; Pereira \& Oliveira, 2018), embora contribua com a melhoria do acesso e qualidade da atenção à saúde, em termos operacionais, ainda constitui um desafio, visto que suas iniciativas ainda são incipientes.

O contentamento expresso nos depoimentos de alguns participantes pode ser consideradas como iniciativas da gestão da clínica, relacionada à cultura de educação que se dá no cotidiano do trabalho em ato, e a supervisão como processo educativo sugere que investir na educação permanente e continuada das equipes é um passo importante para integralidade do cuidado e atuação em redes (Peduzzi \& Agreli, 2018). Essa perspectiva pode se justificar pelo comprometimento e responsabilidade dos enfermeiros líderes deste estudo, a partir de seus relatos na conquista de posicionamento estratégico nos serviços da APS. Esse dado pode ser um fator que contribui para o empreendimento de ações educativas de modo permanente em (re)orientação às práticas de cuidado, sendo relacionado ao construto teórico (Padilha et al., 2018) escolhido para dar luz aos achados. Na tríade dos eixos estruturantes da gestão da clínica, a concepção de educação na saúde é considerada como dimensão importante à sua implementação, pois implica a compreensão de que a aprendizagem ocorre mediante processos de interação social, trocas e relações dialógicas entre sujeitos, com vistas à transformação das práticas.

\section{Considerações Finais}

À luz das falas dos depoentes, as iniciativas de gestão da clínica empreendidas por enfermeiros em posição de liderança na APS são indutoras à implementação de seus princípios, implicando em vantagens que endossam o papel estratégico do enfermeiro na gestão dos serviços de saúde, por impulsionar: a estruturação da assistência em conformidade com as reais necessidades da população, de maneira corresponsável, orientado por melhores padrões de qualidade, e buscando, de maneira colaborativa, a articulação de saberes, práticas e ações educativas permanentes junto aos diferentes atores sociais e pontos de atenção envolvidos no processo de cuidar.

A mobilização em prol de movimentos para a gestão da clínica, tal qual apresentado neste estudo, criou espaço de discussão e estímulo ao protagonismo de enfermeiros em funções estrategicamente importantes na implementação de abordagens propositivas à prestação de cuidados na APS, por meio da clara compreensão e aplicabilidade de seus princípios, uma vez que estes profissionais assumem papel essencial na disseminação e influência de uma cultura favorável à conformação de valores sociais que potencializem qualidade, segurança, corresponsabilização, transparência e integralidade no cuidado. Desta forma, tais iniciativas favorecem a qualificação dos processos de atenção à saúde mediante a transformação das práticas de assistência, gestão e educação.

Cabe aludir que as considerações expostas não permitem fazer generalizações, uma vez que a pesquisa carrega limitações, como o seu recorte, entrevistas individuais e realização em uma realidade particular. Isto posto, sugerem-se estudos mais abrangentes e com múltiplas abordagens metodológicas, a exemplo de pesquisas de natureza aplicada e de caráter transdisciplinar, que favoreçam maior exploração, descrição e explicação de problemas que permeiam a implementação da gestão da clínica, permitindo projetar artefatos voltados à resolução destes no contexto de aplicação.

O estudo é potencializador de discussões que advogam por mais iniciativas e perfis profissionais em posições estratégicas de liderança e que podem resultar na melhoria dos processos de produção de cuidados em saúde das populações, por 
meio de princípios para a gestão da clínica, fornecendo subsídios que possibilitam caminhos propositivos ao equacionamento de desafios de saúde contemporâneos. Considera-se que as alusões apresentadas são, de fato, propulsoras a este movimento no Brasil, já que a clara incipiência sobre o tema elucida oportunidades de maior exploração, domínio e avanço do conhecimento para a área.

\section{Referências}

Anschau, F., Webster, J., Roessler, N., Fernandes, E. O., Klafke, V., Silva, C. P., \& Fossari J. A. J. (2016). Clinic Management as a Tool for Enhancing quality of Care and Guarantee of Hospital Access. BAOJ Medical and Nursing, 2(5), 1-4.

Anschau, F., Webster, J., Roessler, N., Fernandes, E. O., Klafke, V., Silva, C. P., \& Fossari J. A. J. (2017). Evaluation of clinical governance interventions on qualification of care and supply of beds in a large public hospital. Scientia Medica, 27(2), 1-7.

Backes, D. S., Forgiarini, A. R., Silva, L. D., Souza, M. H. T., Backes, M. T. S., \& Büscher, A. (2020). Nursing entrepreneur care in social inequity contexts. Revista Brasileira de Enfermagem, 73(4), 1-6.

Barros, D. F., Freitas, F. R. N., Souza, A. T. S., Araújo, T. M. E., Freitas, D. J. N., Alves, M. C. S., \& Pedrosa, J. I. S. (2021). Liderança do Enfermeiro na Atenção Primária à Saúde: Uma revisão integrativa. Research, Society and Development, 10(1), e26110110099.

Brandt, B., Lutfiyya, M. N., King, J. A., \& Chioreso, C. (2014). A scoping review of interprofessional collaborative practice and education using the lens of Triple Aim. Journal of Interprofessional Care, 28(5), 393-399.

Cassiani, S. H. B., \& Lira Neto, J. C. G. (2018). Nursing perspectives and the "Nursing Now" campaign. Revista Brasileira de Enfermagem, 71(5), 2351-2352.

Cavalcanti, P. C. D. S., Oliveira Neto, A. V. D., \& Sousa, M. F. D. (2015). What are the challenges for the qualification of Primary Care in the view of municipal managers? Saúde em Debate, 39(105), 323-336.

Copelli, F. H. D. S., Oliveira, R. J. T. D., Santos, J. L. G. D., Magalhães, A. L. P., Gregório, V. R. P., \& Erdmann, A. L. (2017). Care management and nursing governance in a maternity ward: grounded theory. Revista Brasileira de Enfermagem, 70(6), 1277-1283.

Cordova, F. P., Santin, J., Barros, A. P. D., Pereira, C. S., Paskulin, L. M. G., Duarte, Ê. R. M., \& Ferreira, G. E. (2020). Artefatos para o cuidado de usuários com lesões crônicas de pele na atenção primária à saúde com base em princípios da gestão da clínica. Research, Society and Development, 9(11), e94991110654.

Dal Molin, T., Oliveira, J. L. C., Tonini, N. S., Oliveira, R. M., Anchieta, D. W., Silva, G. K. T., \& Souza, R. F. (2019). Proactive behavior of hospital nurses: comparison between jobs. Cogitare Enfermagem, 24, e5817.

Ferreira, G. E., Dall'Agnol, C. M., \& Porto, A. R. (2016). Repercussions of proactivity in the management of care: Perceptions of nurses. Escola Anna Nery, 20(3), e20160057.

Galavote, H. S., Zandonade, E., Garcia, A. C. P., Freitas, P. D. S. S., Seidl, H., Contarato, P. C., Andrade, M. A. C. \& Lima, R. C. D. (2016). The nurse 's work in primary health care. Escola Anna Nery, 20(1), 90-98.

Gomes, R., Lima, V. V., Oliveira, J. M., Schiesari, L. M. C., Soeiro, E., Damázio, L. F., Petta, H. L., Oliveira, M. S., Silva, S. F., Sampaio, S. F., Padilha, R. Q., Machado, J. L. M., \& Caleman, G. (2015). The Polysemy of Clinical Governance: a review of literature. Ciência \& Saúde Coletiva, 20 (8), $2431-2439$.

Lanzoni, G. M. M., Meirelles, B. H. S., \& Cummings, G. (2016). Nurse leadership practices in primary health care: a grounded theory. Texto \& Contexto Enfermagem, 25(4), 1-9.

Mattos, J. C. O., \& Balsanelli, A. P. (2019). A liderança do enfermeiro na atenção primária à saúde: revisão integrativa. Enfermagem em Foco, 10(4),164-171.

Mendes, E. V. (2015). A construção social da atenção primária à saúde. Brasília: Conselho Nacional de Secretários de Saúde.

Minayo, M. C. S. (2012). Qualitative analysis: theory, steps and reliability. Ciência \& Saúde Coletiva, 17(3), 621-626.

Oliveira, J. L. C., Toso, B. R. G. O., \& Matsuda, L. M. (2018). Advanced practices for care management: reflections on the Brazilian Nursing. Revista Brasileira de Enfermagem, 71(4), 2060-2065.

Padilha, R. Q., Gomes, R., Lima, V. V., Soeiro, E., Oliveira, J. M., Schiesari, L. M. C., Silva, S. F., \& Oliveira, M. S. (2018). Principles of clinical management: connecting management, healthcare and education in health. Ciência \& Saúde Coletiva, 23(12), 4249-4257.

PAHO. Pan American Health Organization. (2018). Expanding the roles of nurses in Primary Health Care. Washington, DC: PAHO.

Peduzzi, M., \& Agreli, H. F. (2018). Teamwork and collaborative practice in Primary Health Care. Interface - Comunicação, Saúde, Educação, 22(Supl. 2), 1525-1534.

Pereira, J. G., \& Oliveira, M. A. (2018). Nurses' autonomy in Primary Care: from collaborative practices to advanced practice. Acta Paulista de Enfermagem, 31(6), 627-635.

Pinto Filho, H. F., \& Lima, R. R. T. (2020). Gestão participativa em unidades básicas de saúde: uma proposta para reinventar o trabalho em saúde. Research, Society and Development, $9(1), \mathrm{e} 175911872$. 
Research, Society and Development, v. 10, n. 3, e17210313149, 2021

(CC BY 4.0) | ISSN 2525-3409 | DOI: http://dx.doi.org/10.33448/rsd-v10i3.13149

Richter, S. A., Santos, E. P., Kaiser, D. E., Capellari, C., \& Ferreira, G. E. (2019). Being an entrepreneur in nursing: challenges to nurses in a strategic leadership position. Acta Paulista de Enfermagem, 32(1), 46-52.

Santos, J. L. G. D., Erdmann, A. L., Andrade, S. R. D., Mello, A. L. S. F., Lima, S. B. S. D., \& Pestana, A. L. (2013) Nursing governance: an integrative review of the literature. Revista da Escola de Enfermagem da USP, 47(6),1417-1425.

Souza, G. C., Peduzzi, M., Silva, J. A. M., \& Carvalho, B. G. (2016). Teamwork in nursing: restricted to nursing professionals or an interprofessional collaboration. Revista da Escola de Enfermagem da USP, 50(4), 640-647.

WHO. World Health Organization. (2020) State of the world's nursing 2020: investing in education, jobs and leadership. Geneva (SUI): World Health Organization. 